\title{
Machine Vision based Color Recognition by Robotic Arm using LabVIEW
}

\author{
Y. Divya ${ }^{1}$ and C. Pramod Kumar ${ }^{2}$ \\ ${ }^{1}$ Asst. Professor, CVR College of Engineering/EIE Department, Hyderabad, India \\ Email: divya.reddy037@gmail.com \\ ${ }^{2}$ Asst. Professor, CVR College of Engineering/EIE Department, Hyderabad, India \\ Email: pramod.kumar@cvr.ac.in
}

\begin{abstract}
Machine vision-based color recognition by robotic arm using LabVIEW, which detects a particular color through webcam fixed in robotic arm using LabVIEW and image processing is set forth in this paper. Color recognition allows robotic arm to detect a particular color that is selected in the front panel of LabVIEW. As soon as the particular color gets identified, it collects the selected color and after some time it is placed back to its original position. The advantages of this method include the reduction of errors and the time over manual labor. And robotic arm's reliability is more.
\end{abstract}

Index Terms: Image processing, LabVIEW, color recognition, Arduino Uno

\section{INTRODUCTION}

The aim of this paper is to control robotic arm to perform various applications like movement of robotic arm, color recognition of objects using LabVIEW[13]. The industrial robotic arm can be controlled by various means such as microcontrollers, visual basic and etc. These have certain drawbacks such as code-compilation, complex programming. To overcome these drawbacks National instruments LabVIEW [13] software is used. [7].

LabVIEW utilizes virtual instrumentation platform, which works on customized software and modular measurement hardware [13]. User defined measurement system is created, signals and graphical coding are generated by using LabVIEW [12]. This kind of robotic arm in industries can be controlled by the non-programmers too.

The main challenge is to improve the existing tracking system in the modular processing, containing 4 stations as identification, processing, selection and display with a new image processing feature. The present tracking method utilizes set of inductive, capacitive and auto sensors in identifying the object color[12].

Color identification system controlled by LabVIEW [13] through image processing [12] is presented in this paper. Objects are captured by webcam in real-time mode through Image processing [12] the color and information details are detected. Information is processed through image processing technique [12] for pick - and - place operation. The apparatus learns to identify objects based on a two - phase operative methodology which is defined as autodidactic stage; An operative choice manner wherein items are detected, categorized the use of a decisional set of rules and decided on in real-time [11].

This work deals with an automated robotic arm controlled by LabVIEW[11]. Thereby removing the monotonous work finished through human, to achieve faster and accurate work. Objects color is captured through webcam and will send the signal to the Arduino board [12].

LabVIEW receives signals from Arduino which drives different models of the robotic arm to track the colors of the objects. Depending on the robotic detection, robot arm will be moved to the specified vicinity, releases the object and will be returned back to its original position.

\section{Robotic Arm InTERFACED WITH ARduINo}

L293D motor drives robotic armwith 5 automobiles and those vehicles. L293D is a standard Motor driving force [7] or Motor Driver IC [7], which permits DC motor to run on both routes. L293D is 16 - pin IC which is able to control a set of two DC automobiles concurrently in any course. It means that it may be manipulated by DC motor with L293D IC [7]. L293D can run small and quiet huge motors as well. The cars are connected to these drivers in keeping with their automobiles, respectively.

A Magnetic Field Generating coil is placed in the filed winding for getting the motor action i.e. whenever the magnetic field is generated due to turning on and off the modem or due to switching the path of the modem within the coil. A Dc Motor has restricted magnetic field inside the stator and an armature with a sequence of extra wrapped winding in mica insulated stack slots around iron pole portions (known as stack teeth) whose ends terminating on a Mechanical Converter of DC to AC. With the assist of these motors the robotic arm action accordingly.

Now the robotic arm is interfaced with the Arduino board [14] which has a microcontroller board based on ATmega328 (datasheet)[14]. It has 14 virtual enter/output pins (out of which 6pins can be used as PWM outputs)[14], 6 analog inputs, a $16 \mathrm{MHz}$ crystal oscillator, a USB connection, an energy jack, an ICSP header, and a reset button. It includes a microcontroller, connected to a PC with USB cable or external power supply, with an AC-to-DC 
adapter or battery to get started[14]. The Arduino Uno and model 1.0 may vary from eachother in transferring ahead. The Uno is a series of USB Arduino forms[14]. As the signals from a laptop are shipped to the arduino board thet are sent as alerts to the robot arm to go as directed.

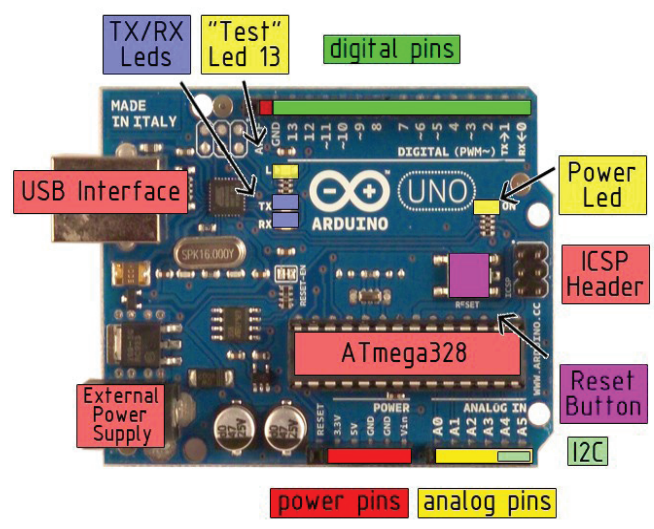

Figure 1. Technical specifications of Ardiuno board

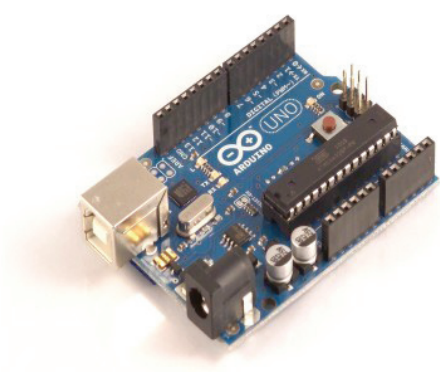

Figure 2. Arduino board

\subsection{Usage of Arduino}

Environment is sensed by a kind of sensors and people indicators are sent to Arduino[14] which is a good way to range its environment with the aid of controlling lighting fixtures, automobiles, and other actuators. It consists 14 virtual input/output pins[14] (out of which 6pins may be used as PWM outputs), 6 analog inputs, a $16 \mathrm{MHz}$ crystal oscillator, a USB connection, an power jack, an ICSP header, and a reset button[14]. It includes a microcontroller is which connected to PC with a USB cable or with an ACto-DC adapter/battery to get started. The Uno differs from all previous forums where the FTDI USB-to-serial is not used [14]. Different instructions are to be followed according to individual OS.

\subsection{Requirements}

1. Arduino Duemilanove board

2. USB Cable

3. Nine volt battery or external power supply (for nonredundancy operation)

4. Solderless breadboard for external circuits, 22 solid wire connections.
5. Host PC running the Arduino development environment, Windows, Mac and Linux have their own versions.

\section{POWER SUPPLY}

The board is powered through USB Connection with the computer. The external power supply is given in the range of 6 to 24V. A fashionable nine volt Battery [14] can be connected to the board by connecting the leads of a battery to Vin pin and Gnd pin. Soldering is best option to connect battery ends in a DC plug and electricity jack at the board, by taking an appropriate plug component from the wide variety available under 28760 .

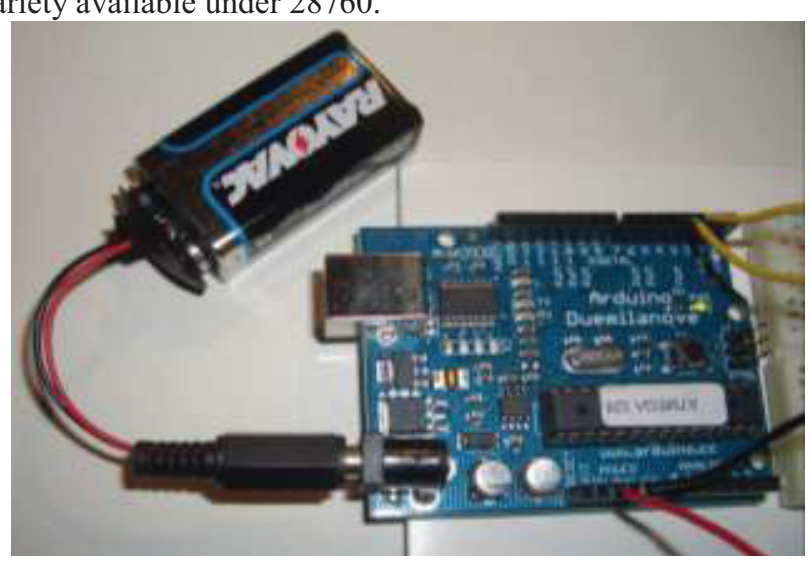

Figure 3. Connecting Battery to Arduino board

Polarity need to be checked while connecting the battery, as to avoid blow down of the board.

Arduino should be disconnected from the computer and Nine volt battery should be connected to the Arduino power jack using the snap battery adapter. The blinking light confirms its active state. This means the Arduino can be powered through a battery and need not be defined on having a connection to the host PC. Arduino can be connected with the computer by using USB cable [14], the green LED will glow. It will run the code if already exists in the board.[14].

Precaution to be taken: Board should not be placed on a conductive floor because pins on the backside of the board may get short circuited.

Arduino development environment can be commenced. In Arduino-speak, programs are called "Sketches", but right here they're just known as packages[14].

In the modifying window that comes up, input subsequent program taking note of in which semi-colons seen on the command strains.

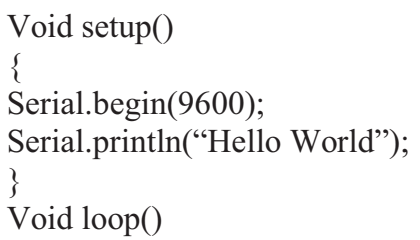


\{\}

Your window will look something like this[14]

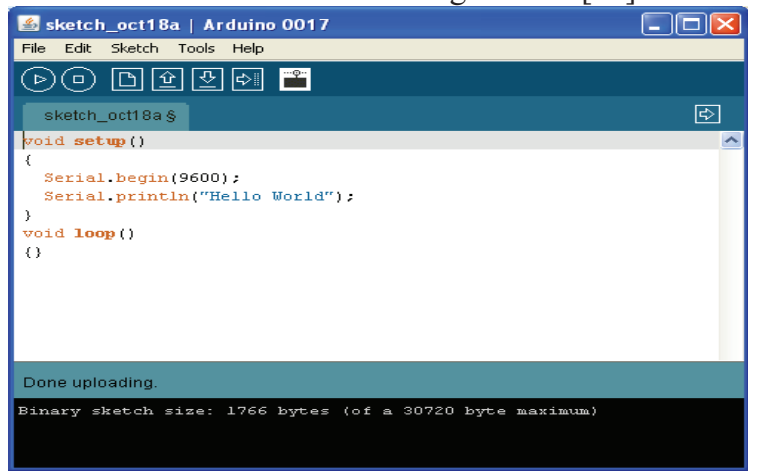

Figure 4. Write code instruction Screenshot

Upload button need to be clicked $\Rightarrow$ or to compile the program Ctrl-U and later load on the Arduino board.

Select serial monitor button. If there are no errors, the serial monitor window will display message as below.

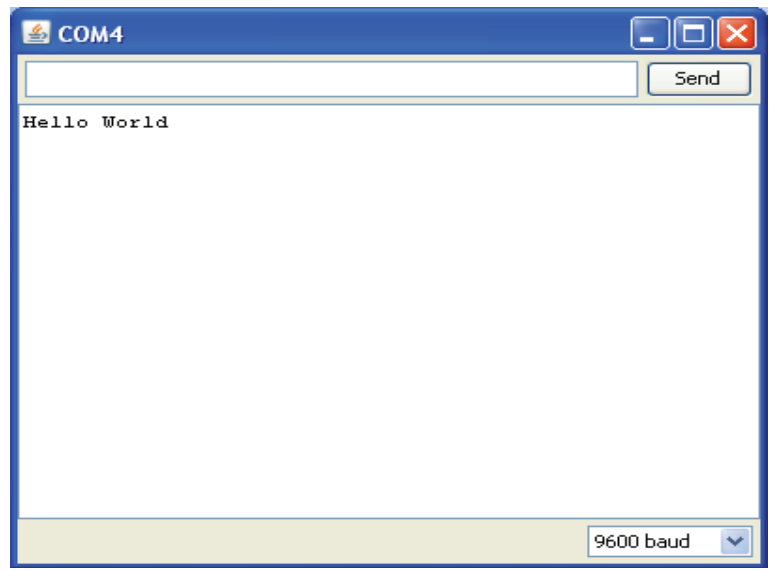

Figure 5: Displayed output

\section{Digital Image MAThematical INTERPRETATION}

Image is handled as a matrix of MxN factors [12]. Each element of the digitized picture (pixel)has a price that corresponds to the brightness of the factor inside the captured scene. An Image whose decision in depth is of 8 bits, can take values from zero to 255.In the case of a black and white photograph it may take 0 and 1 values. In general picture is represented in a bi-dimensional matrix.

Maximum number of devices collects the pictures with an eight bit intensity. The ranges of gray for a picture[4]is from zero to two hundred and fifty five in order of the matrix elements of the image are represented by using xij $[0 \ldots 255]]$. At this point it's far handy to say that, despite the fact that the photographs are received in RGB layout, it is frequently converted into a gray scale matrix, for achieving the transformation from RGB form to Grassmanstage(Wyszeeki\&Stiles, 1982)isemployed:Igray=I
$\mathrm{R}(0.299)+\mathrm{IG}(0.587)+\mathrm{IB}(0.114)[7]$. In the example presented in Fig.4, a way to collect a virtual photograph in RGB format is suggested and grayscale layout the usage of the IMAQ toolbox [13].

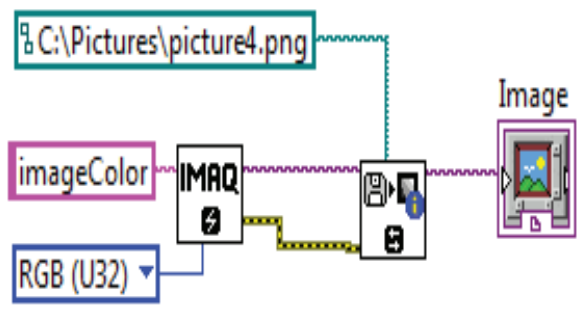

Figure 6. IMAQ Create

In this situation, there are crucial blocks: The first one is the IMAQ create block located in Vision and Motion/Vision Utilities/Image Management. This block creates [13] grayscale, HSL, and so on.), while the second block is the IMAQ Read Image which is placed in vision and movement/imaginative and prescient utilities/documents, the characteristic property of this block is to open picture file that was precisely formed in document route. All records of this opened photograph are placed in the new photograph created through IMAQ create [7]. In different form this instance is presented in Figure. 6 and the record Figure. 6 is opened as IMAQ Read Image. The facts of image are saved in a brand new photograph called image color. It is related to $\mathrm{RGB}(\mathrm{U} 32)$ photograph format of the gadget. In Figure.7 the picture kind is changed to Grayscale(U8) and the picture is placed in image Gray.

Another crucial feature within the picture is defined by neighborhood pixel which is classified in to 3 agencies described in Figure.6. If the neighborhood is limited to 4 adjoining pixels, it is only confirmed by diagonal pixels is the D-Neighborhood and eight surrounding pixels is eightneighborhood, the remaining consist of Four and Dneighborhood.

\section{Histogram IMAge}

Histogram image is a graph which contains number of pixels in an image at different intensity values. In Eight-bit gray scale showing the distribution of pixels with gray scale values as shown below [13].

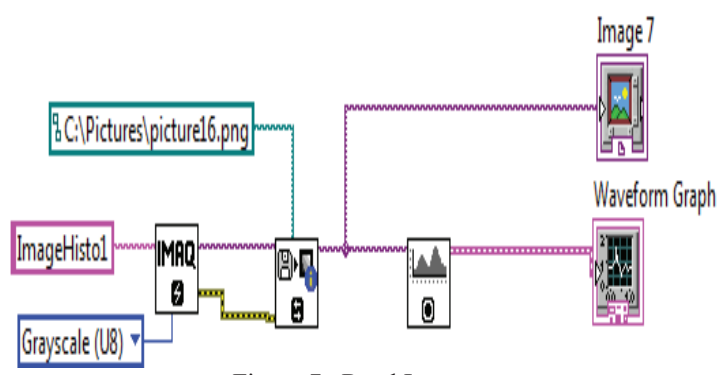

Figure 7. Read Image 
When it is seen through histogram gray scale, it appears as the below given Figure 8

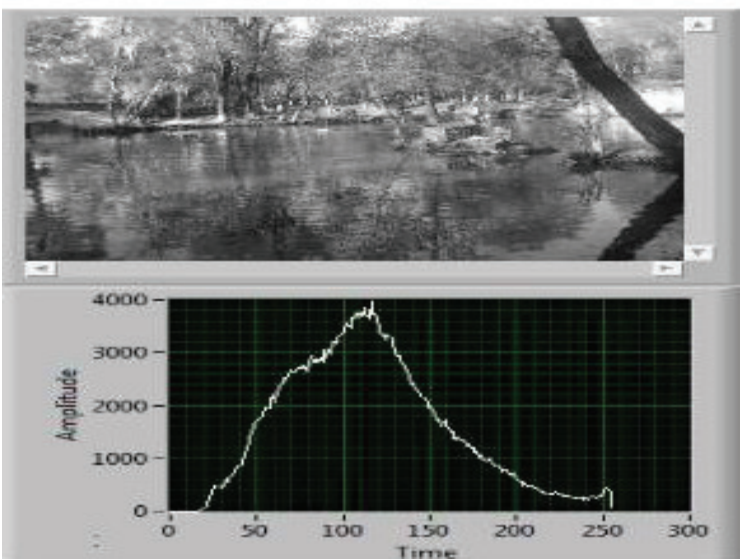

Figure 8. Histogram gray scale

The above shown are the output of grayscale histogram

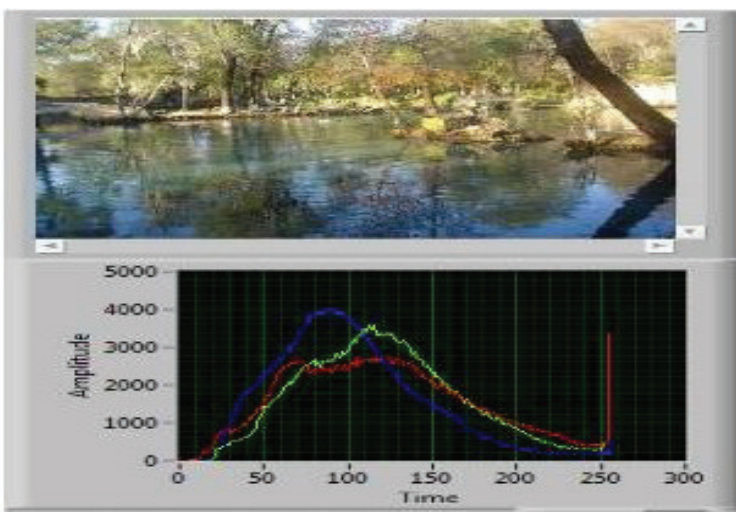

Figure 9. Histogram RGB image

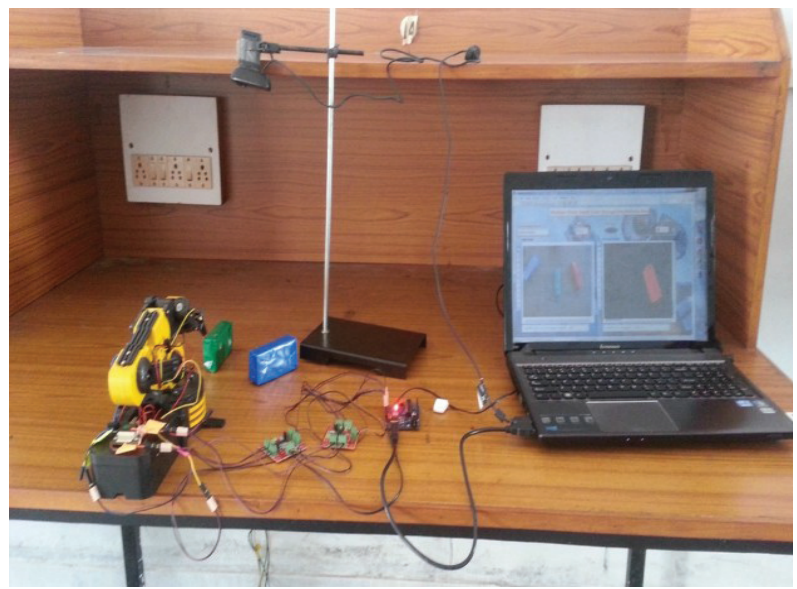

Figure 10. Robotic arm complete Setup
CVR Journal of Science and Technology, Volume 18, June 2020

DOI: $10.32377 /$ cvrjst1817

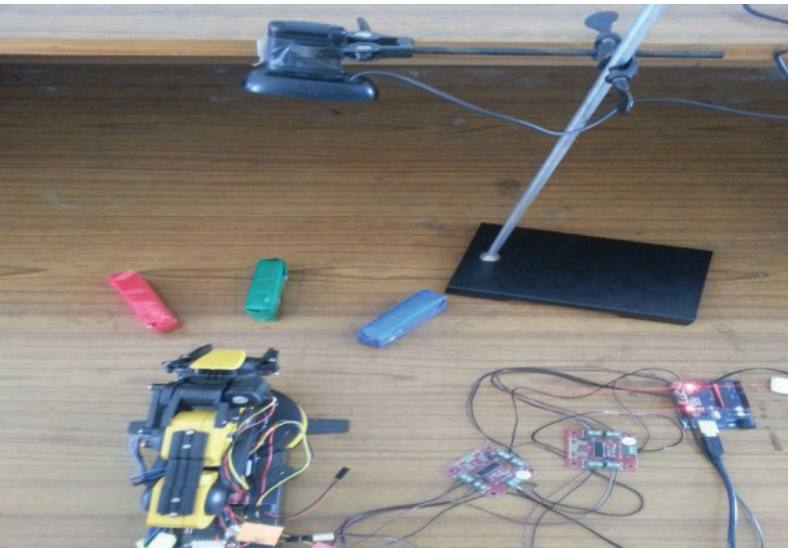

Figure 11. Image Capture through Webcam

image and RGB histogram image using [1] IMAQ histogram image Image Processing [12]. The output of the IMAQ histogram image is a waveform graph, which is connected to show obtained results.

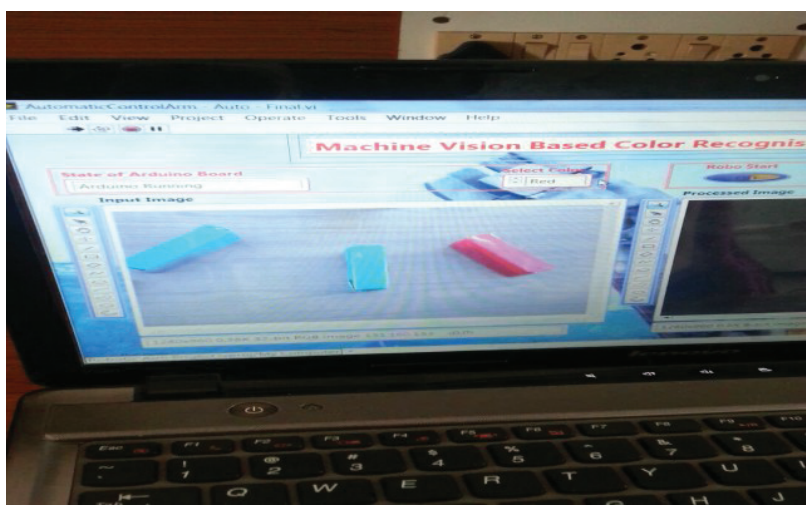

Figure 12. Input View in Front Panel

\section{RESULT}

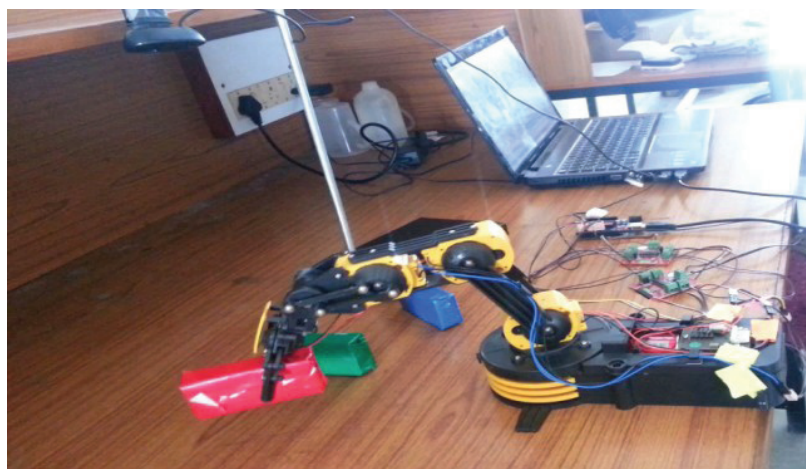

Figure 13. Operation of Robotic arm

The image of the particles is captured by the webcam connected to the retard stand. After the image is captured by the webcam in front panel the input looks in this way. As it detects the image the robotic arm automatically picks that particular object [2] and places it back to its original position. 


\section{CONCLUSIONS}

Hence it is concluded from the above points is the man ability and arduous effort can be reduced using the robotic arm controlled by LabVIEW by picking and placing the objects. The robotic arm designed above is used to track the prescribed color among the different colors [10].The controlling software is also very convenient for the nonInstrumentation engineers also.

\section{REFERENCES}

1. M. Swain, and D. Ballard, -Color indexing\|, International Journal of Computer Vision, Vol No. 7, pp. 11-32, 1991.

2. B.Schiele and J. 1. Crowley, -Recognition without correspondence using multidimensional receptive field histograms\|, International Journal of Computer Vision Vol. 36, pp. 31-50, 2000.

3. H. Schneiderman, and T. A. Kanade, -Statistical method for $3 \mathrm{~d}$ object detection applied to faces and carsll, IEEE Computer Society Conference on Computer Vision and Pattern Recognition, Vol 1, pp. 1746, 2000.

4. S. Agarwal, A. Awan and D. Roth, - Learning to detect objects in images via a sparse, part-based representationll, IEEE Transactions on Pattern Analysis and Machine Intelligence, Vol. 26, pp. 1475 - 1490, 2004.

5. A. A. Ata, A. Rafeek and H. Yusof, -Sensory-Based color sorting automated robotic celll, Journal of Intelligent and Robotic Systems, Vol. 1, pp. 99-110, 2005.

6. Junqiu Wang and Yasushi Yagi, - Integrating Color and ShapeTexture Features for Adaptive Real-Time Object
Tracking \|, IEEE Transactions On Image Processing, Vol. 17, pp. 235-240, 2008.

7. P.B.Vijayalaxmi, Rohan Putta, Gayatri Shinde, Punit Lohani, -Object Detection Using Image Processing For An Industrial Robotll, International Journal of Advanced Computational Engineering and Networking, Vol.1, pp.21-26, 2013

8. Binbin L., Yimin S., Gang D., Tao S., Yang Q., —Dimensional Synthesis of a Planar Parallel Manipulator for Pick-and-Place Operations Based on Rigid-Body Dynamics, Intelligent Robotics and Applications/Lecture Notes in Computer Science, Springer, Vol. 7506, pp. 261-270, 2012.

9. Altuzarraa O., Şandru B., Pinto Ch., and Petuya V., -A Symmetric Parallel Schönflies-Motion Manipulator for Pickand-Place Operationss, Robotica, Vol. 29, Issue 06, pp. 853- 862, October 2011.

10. Wenjing Li, George Bebis and Nikolaos G. Bourbakis (2008) -3-D Object Recognition Using 2-D Viewsll, IEEE transactions on Image Processing, Vol. 17(11), 2236-2255.

11. Adrian D. Olaru, Serban A. Olaru, Niculae F. Mihai, and Natalia M. Smidova-Animation in Robotics with LabVIEW Instrumentation, International Journal of Modeling and Optimization, Vol. 9, 2019.

12. Thomas Klinger, Image Processing with LabVIEW and IMAQ Vision, Prentice Hall Professional, 2003.

13. National Instruments, LabVIEW for color Recognization, 2019.

14. Leo Louis1, Working Principle Of Arduino And Using It As A Tool For Study And Research, International Journal of Control, Automation, Communication and Systems (IJCACS), Vol.1, No.2, April 2016. 